\title{
Non-Invasive Approaches for Oral Potential Malignant Disorders Surveillance: A Review
}

\author{
Jiaying Zhou1,2*, Quan Liu' ${ }^{2,3 *}$, Xianyue Ren², Bin Cheng1,2, Tong Wu ${ }^{1,2 \#}$ \\ ${ }^{1}$ Department of Oral Medicine, Affiliated Hospital of Stomatology, Guanghua School of Stomatology, Sun Yat-sen University, \\ Guangzhou, China \\ ${ }^{2}$ Guangdong Provincial Key Laboratory of Stomatology, Guanghua School of Stomatology, Sun Yat-sen University, Guangzhou, \\ China \\ ${ }^{3}$ Zhujiang New Town Dental Clinic, Affiliated Hospital of Stomatology, Guanghua School of Stomatology, Sun Yat-sen University, \\ Guangzhou, China \\ Email: "wutong23@mail.sysu.edu.cn
}

How to cite this paper: Zhou, J.Y., Liu, Q., Ren, X.Y., Cheng, B. and Wu, T. (2020) Non-Invasive Approaches for Oral Potential Malignant Disorders Surveillance: A Review. Advances in Bioscience and Biotechnology, 11, 188-205.

https://doi.org/10.4236/abb.2020.115015

Received: April 12, 2020

Accepted: May 19, 2020

Published: May 22, 2020

Copyright $\odot 2020$ by author(s) and Scientific Research Publishing Inc. This work is licensed under the Creative Commons Attribution International License (CC BY 4.0).

http://creativecommons.org/licenses/by/4.0/

\begin{abstract}
Oral potential malignant disorders (OPMDs), a series of local diseases with carcinogenic potential occurred in oral mucosa, have the possibility of developing into oral cancer. Effective diagnosis and surveillance at the early stage of OPMDs may be of help for interrupting the malignant transformation. In this review, we introduced the available non-invasive approaches of detecting the suspicious lesions, including vital staining, oral cytology, optical detection, saliva biomarkers detection, and image analysis, which can enhance the detection efficiency for the early diagnosis and surveillance of OPMDs. However, limitations which restrict the clinical application of these approaches still exist. How to improve the sensitivity and specificity of these techniques deserves further detailed study.
\end{abstract}

\section{Keywords}

Oral Potential Malignant Disorders, Non-Invasive Detection Approaches, Malignant Transformation, Early Diagnosis, Surveillance

\section{Introduction}

Oral potential malignant disorders (OPMDs) are diseases with potential carcinogenesis in oral mucosa, which have evidently clinical and histological alternations, such as leukoplakia, erythroplakia, oral submucous fibrosis, lichen plaus and discoid lupus erythematosus. It was reported that approximately $16 \%-62 \%$

${ }^{\star}$ Jiaying Zhou and Quan Liu are the first authors and contribute equally to this work. 
of epithelial dysplasia cases of OPMDs underwent malignant transformation and eventually developed into oral squamous cell carcinoma (OSCC) [1]. OSCC is the most common oral cancer, accounting for more than $90 \%$ of all oral cancers. In the past few decades, the five-year survival rate of OSCC patients in the early stage is about $80 \%$, while it rapidly decreased to $40 \%$ in the advanced stage. As early diagnosis and timely monitoring substantially reduce the cancer-specific morbidity and mortality in OSCC, the surveillance of OPMDs is of great significance for the prevention of OSCC [2].

Although the pathological examination is the gold standard for the diagnosis of OPMDs, it is invasive to obtain pathological tissue through surgical operation, which is hardly acceptable by patients. Furthermore, such surgical processes involve certain risks including postoperative scars and excessive bleeding, so that they are not suitable for multiple operations for the early diagnosis and long-term monitoring of OPMDs [3]. Currently, non-invasive detection methods for early diagnosis, such as vital biological dye staining, oral cytology, optical detection and image diagnosis have been widely used in clinical practice. These methods seem to replace tissue biopsy because of their efficiency and accuracy, as well as the improved acceptance of patients [4]. The purpose of this review is to summarize the current non-invasive diagnostic techniques for the monitoring of the malignancy of OPMDs, which may effectively lower the occurrence of oral cancer.

\section{Vital Staining Techniques}

\subsection{Toluidine Blue Staining}

Toluidine blue (TB), a common cationic metachromatic alkaline dye, has been used as an important stain to highlight oral potential malignant lesions since the early 1980s. Cells in dysplastic or malignant tissue are more disordered in arrangement, looser in connection and contain more nucleic acid than normal cells. Therefore, TB can enter the extracellular space in dysplastic or malignant tissue, and bind to the intracellular nucleic acid to make the tissue blue, which can be readily distinguished [5].

It was reported that the sensitivity and specificity of TB staining in detecting high-risk pre-cancer lesions were $94 \%$ and $45 \%$, respectively, whereas for carcinoma, the data were $100 \%$ and $39 \%$. Therefore, TB staining combined with clinical examination may help monitor the development of OPMDs and identify severe dysplasia or cancer [6]. Besides, the operation of TB staining is simple and its result is easy for observation. However, the drawback of TB staining is the possibility of false results. For instance, the space between epithelial cells in inflammatory lesions can be enlarged, and TB could also stain neutrophils and bacterial nuclear fragments, which lead to false positive results. On the contrary, there is no nucleus in the stratum corneum for the epithelium with hyperkeratosis, which can be hardly stained by TB and may result in false negative results [7]. 


\subsection{Lugol's Iodine Staining}

Lugol's iodine staining was firstly used to assist the diagnosis of early esophageal cancer in 1770s, and applied in the early screening of cervical, oral and some other cancers afterwards. The underlying mechanism of Lugol's iodine staining depends on its specific interaction with the glycogen in the cytoplasm. As the content of glycogen in the cytoplasm of normal oral mucosal tissue is higher than that in the dysplastic and malignant tissue, the color of the two kinds of tissue is different after staining [8]. Specifically, the normal mucosal tissue turns brown, while the dysplastic and malignant tissue becomes white or light yellow due to the active metabolism and significantly reduced glycogen. Thus, as a simple, fast, non-invasive and inexpensive method, Lugol's iodine solution staining can be used to indicate the lesion sites and better delineate their boundary.

The clinical accuracy of Lugol's iodine was reported to be $76 \%$ in detecting dysplasia of OPMDs from other benign mucosal lesions, indicating its value in the detection of dysplasia and malignant transformation of OPMDs. However, false results might be observed. On one hand, the content of glycogen in epithelial cells is positively correlated with the differentiation degree of tumor cells. The glycogen content in mild epithelial dysplasia and highly differentiated squamous cell carcinoma was higher than that in severe dysplasia and lowly differentiated cancer cells, resulting in false negative results in the former two when stained with Lugol's iodine. On the other hand, false positive results may occur in benign lesions with thick keratinizing layer because of the decreased glycogen [9].

As TB staining shows a high false positive rate while Lugol's iodine staining has a high false negative rate, it was recommended that a combination of them may improve the detection accuracy up to $90 \%$ [10].

\subsection{Rose Bengal Staining}

Rose Bengal (RB) is a derivative of luciferin. The main staining mechanism of $\mathrm{RB}$ is its special affinity to DNA polymerase. RB dye binds DNA polymerase together in the form of ionic bond to stain the nucleus. RB also can strongly bind to the nuclear histones and other organelles [11]. The contents of nucleic acids and DNA polymerases are abnormally increased in dysplastic and malignant cells due to its rapid proliferation, resulting in different absorptions of RB in patients with OPMDs and OSCC. Therefore, RB staining was considered to assist the early diagnosis of precancerous malignant change and OSCC. It was found that the intensity of RB staining was correlated with dysplasia grades, indicating its application in identifying normal tissue, simple hyperplasia, mild, moderate and severe dysplasia. Nevertheless, false positive results might be caused by the inflammatory lesions, which are easier to be stained by RB [12].

\section{Cytological Techniques}

The process of cytological techniques is divided into two steps. The first step is cell collection from the oral mucosal surface, including traditional exfoliative cell 
collection and oral cell brush technique. The second step is cytological diagnosis or biomarker detection. Cytological diagnostics will enhance the ability to detect individual risk, while analysing extracted material allows for more sensitive and specific techniques.

\subsection{Cell Collection}

\subsubsection{Traditional Exfoliative Cell Collection Technique}

In the past few decades, the most commonly used traditional tools for collecting oral mucosa cells were metal spatula, wooden tongue depressor and cotton swab. The truth is that the sample cells collected with these traditional tools are insufficient for further detection and analysis, which may cause a high false negative rate. Furthermore, metal spatula could induce trauma and infection, which is hardly acceptable to patients [13].

\subsubsection{Oral Brush Cytological Technique}

Compared with the traditional cell collection technique, the oral brush cytological technique can collect the whole layer of oral epithelium as well as the emerged basal cells. OralCDx system (OralCDx Laboratories, Suffern, NY, USA) is a kind of computer aided system along with a special oral brush for the collection of transepithelial sample. With OralCDx, pathological diagnosis could be made according to whether and how much the cells have atypia through its microscopic analysis by computer imaging system. Moreover, the collected cells can be detected with the relevant biomarkers to evaluate the carcinogenesis risk of oral mucosal lesions [14]. The Orcellex ${ }^{\circledR}$ brush (Rovers Medical Devices B.V., Oss, The Netherlands) is the latest cell brush customized for the use in mouth, with a high efficacy of $99 \%$ smears with adequate cells. Nevertheless, in the cases of ulceration, hyperemia, necrosis or excessive bleeding in the lesion sites, it may become difficult to obtain valuable specimens and lead to missed diagnosis [15].

\subsection{Contents Detection}

\subsubsection{Cell Morphology Detection}

The collected cells can be made into smears for cytological diagnosis. Oral liquid-based cytology (OLBC) is characterized by displaying the cell morphology and staining for the instant fixation of cells while removing unwanted substances [16]. High feasibility and effectiveness of OLBC with oral brush samples have been reported. The detection specificity, sensitivity, positive predictive value (PPV), negative predictive value (NPV) and accuracy rate of OLBC with brush biopsy of Orcellex ${ }^{\circledR}$ were found to be $76 \%, 75 \%, 76 \%, 75 \%$ and $75 \%$, respectively [17]. It was also reported that OLBC with clinical oral brush biopsy was simpler and more efficient than conventional cytology. The sensitivity, specificity, PPV and NPV of the cytological diagnosis were $97.5 \%, 68.8 \%, 88.76 \%$ and $91.7 \%$, respectively, which may improve the diagnostic accuracy of oral brush biopsy in the future [18]. 


\subsubsection{DNA Content/Ploidy Detection}

DNA is the basis of cell growth, differentiation and proliferation. Genetic alterations caused by various carcinogenic factors lead to DNA content variation which was mainly detected by DNA flow or DNA-image cytometry on stained nucleus of cells obtained from clinical brush samples from OPMDs [19]. Aneuploidy refers to the number abnormality of DNA, which is represented with DNA index (DI), i.e. the ratio of the DNA content in the sample cells to that in the normal diploid cells. Aneuploidy with a presence of $20 \%-92 \%$ in the patients with oral epithelial dysplasia is often taken as a specific indicator of malignant transformation and carcinogenesis [20]. Also, it was found that the DI of the normal diploid cells was about $0.85-1.15$ and increased significantly in dysplastic and malignant cells [21].

Compared with the autofluorescence technique, DNA-image cytometry combined with brush biopsy to detect the DNA content showed a higher accuracy in diagnosis in terms of sensitivity (93.51\%), specificity (90.10\%), PPV (79.12\%) and NPV (97.19\%) [22]. Such a high diagnostic accuracy was also found in other reports where DNA-image cytometry and cell brush were used together. The sensitivity, specificity, PPV and NPV of such a combined use in the early diagnosis of OPMDs carcinogenesis were $86.36 \%, 90 \%, 86.36 \%$ and $90 \%$, respectively. Hence, DNA content analysis technique may not only provide basis for the early diagnosis of OPMDs, but also play an important role in monitoring the malignancy in oral mucosa [23] [24].

\subsubsection{Copy Number Aberrations Detection}

Copy number aberrations (CNAs) refer to the abnormal deletions and duplications in the genome, which are associated with malignant transformation of OPMDs. CNAs can be analyzed by the array comparative genomic hybridization (aCGH), single-nucleotide polymorphism technique (SNP), and next generation sequencing (NSP) from extracted DNA [25].

Some specific CNAs have shown a high expression frequency of $40 \%-60 \%$ in OPMDs. It was reported that the average of CNAs was 1.9 in non-dysplastic mucosa and 6.5 in OPMDs. Besides, the gains of 20q13.31 - q13.33 and 5 p13.33-pter, and the loss of 9p21.3 occurred more frequently in patients with OPMDs than the control, at frequencies of $62.5 \%, 50 \%$ and $50 \%$, respectively [26]. Some studies manifested that the gains in 3q, 5p, 7p, 8q, 11q and 20q, and the losses in $3 p, 8 p, 9 p$ and $18 q$, were the most commonly observed chromosome aberration regions [27].

\subsubsection{Micronucleus Detection}

Micronucleus is a centerless chromatid, or chromosome fragment, formed after genetic damage caused by toxic substances contained in tobacco and betel nut. The newly formed cells of oral epithelium replace the exfoliative cells as they migrate to the epithelium surface. Thus, the genetic damage of the basal cells can be reflected by detecting the material of nucleus after staining the smear of exfo- 
liative cells [28]. It was found that micronuclei count increased significantly during the transformation of normal mucosa to OPMDs, which indicated that micronucleus can be used as a marker for the diagnosis of OPMDs. Besides, micronucleus detection of exfoliated epithelial cells was found to be a good biomarker for detecting chromosome loss or mitotic spindle dysfunction [29].

\section{Optical Detection Techniques}

The optical detection system has special light sources, which can be absorbed and reflected by abnormal and normal tissue of oral mucosa with different degrees. Currently, chemiluminescence techniques, such as ViziLite and ViziLite Plus emit chemiluminescent light to visually identify lesions, and autofluorescence techniques, including Visually Enhanced Lesion scope (VEL scope) and Identafi induce tissue fluorescence to detect suspicious tissue, both of which are commonly used in optical detection of OPMDS and oral cancer (Figure 1).

\subsection{ViziLite}

As firstly introduced in 2001, ViziLite (Zila Pharmaceuticals, Phoenix, AZ, USA) was used to detect lesions by using a hand-held, disposable chemiluminescent rod emitting light. ViziLite Plus (Zila Inc., Ft Collins, CO, USA) is a combination of a chemiluminescent light source (ViziLite) to identify OPMDS abnormalities and an active tissue dye (toluidine blue) to mark them simultaneously. With ViziLite/ViziLite Plus, the absorption and reflection of light by the mucosal tissue with metabolic abnormalities or structural changes are different from that by the normal tissue. Normal epithelial cells absorb the light and become dark, while hyperkeratosis and abnormal hyperplasia lesions turn white [30].

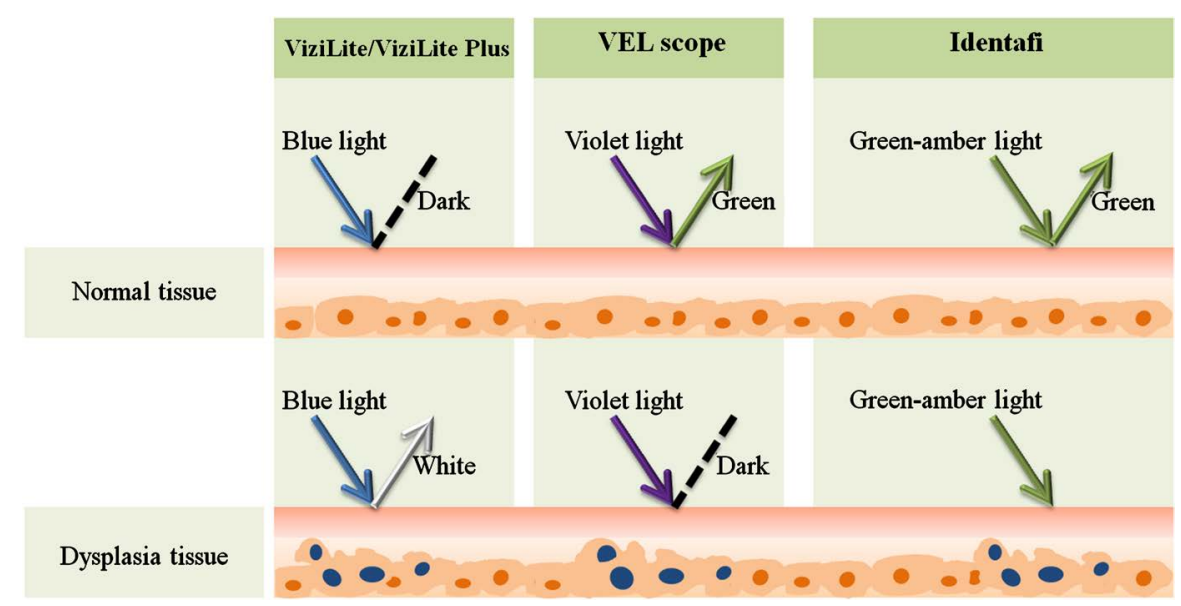

Figure 1. Comparison of different optical detection systems for the non-invasive examination of dysplasia tissue (data from authors). A: ViziLite or ViziLite Plus: Normal tissue appears dark by absorbing chemiluminescent light while dysplasia tissue looks white by reflecting it. B: VEL scope: Normal tissue with endogenous autofluorescence substances looks green under the 400 - $460 \mathrm{~nm}$ violet light while dysplasia tissue turns dark because of weakened autofluorescence. C: Identafi: Normal tissue reflects green light whereas dysplasia tissue exhibits increased vascularity under green-amber light. 
As reported, the ViziLite showed a diagnosis accuracy of $100 \%$ sensitivity, $30 \%$ specificity, $26 \%$ PPV and 100\% NPV for the low specificity malignancies. Vizilite Plus identified both serious and benign abnormalities in the OPMDs and inflammation cases with sensitivity, specificity, PPV and NPV of $80 \%, 97.5 \%$, $89 \%$ and $95 \%$, respectively [31]. In the comparison of sensitivity and specificity between Vizilite and toluidine blue, the former was found to be more sensitive but less specific than the latter. Compared with Vizilite Plus with a sensitivity of $100 \%$, specificity of $97.3 \%$, PPV of $100 \%$ and NPV of $75 \%$, Vizilite is more effective but less specific for detecting abnormal hyperplasia [32]. What should be mentioned is that the color difference of oral mucosa caused by Vizilite and/or Vizilite Plus may be related to epithelial thickness, which may lead to misdiagnosis. In addition, Vizilite was found to improve the visibility and clarity of white lesions, especially for oral leukoplakia, which can effectively distinguish white spots from normal tissue, but for the observation of red spots, the distinguishing ability dropped to $50 \%$. Therefore, the effect of this system in detecting red lesions remains to be further studied [33].

\subsection{VEL Scope}

Visually Enhanced Lesion scope (VEL scope, White Rock, BC, Canada) is a set of simple-operating equipment with the application of autofluorescence technique for OPMDs identification. Specifically, VEL scope emits violet light with a wavelength of $400-460 \mathrm{~nm}$. Stimulated by the light, the normal mucosa becomes pale green, while the dysplasia and malignant lesions turn darker than the surrounding healthy tissue due to the weakened autofluorescence [34]. The underlying mechanism depends on the changes in the distribution and structure of fluorophore groups in the epithelial cells and subepithelial matrix in the abnormal tissue. For example, the alteration or destruction of collagen and elastin may lead to the loss of autofluorescence and show different colors from the normal tissue under VEL scope [35].

VEL scope is a simple and non-invasive method to detect and help locate malignant oral diseases, which could be used as an important auxiliary monitoring approach for high risk oral lesions. For the application of VEL scope in the diagnosis of OPMDs, the sensitivity, specificity and accuracy were $94.4 \%, 96.2 \%$ and $96.1 \%$, respectively. As for epithelial dysplasia detection, the sensitivity, specificity and accuracy of VEL scope were $100 \%, 92.4 \%$ and $92.6 \%$ [36]. Compared with iodine staining, the positive rate of epithelial dysplasia detected by VEL scope was significantly higher, indicating optical fluorescence imaging is an important tool to identify OPMDs with dysplasia [37].

However, the increase of hemoglobin in inflammatory hyperemia site enhances the absorption of light and leads to fluorescence deficiency, which may cause false positive result of VEL scope. Moreover, false negative result might be obtained when the keratinization degree of the mucous membrane is high. Thus, the effect of this technique to distinguish abnormal hyperplasia from benign le- 
sions needs further observation [38].

\subsection{Identafi}

Angiogenesis is a known process in the progression of OPMDs and oral cancer. The vascular activity in the dysplasia and malignancy tissue is more active than that in the normal tissue, which can be detected by Identafi device (DentalEZ Inc, Malvern, PA, USA). Identafi possesses two Light Emitting Diodes (LEDs) which can generate lights with three wavelengths (white, violet and green-amber light) to observe tissue fluorescence and reflectance. In addition to the similar function of VEL scope, the green-amber light (wavelength 540 - $575 \mathrm{~nm}$ ) can reveal the abnormal angiogenesis in the lesion sites, as the increased microangiogenesis and oxyhemoglobin concentration in these sites enhances light absorption and reduces tissue reflectivity. Identafi showed a sensitivity of $82 \%$ and a specificity of $87 \%$ in distinguishing tumor from non-tumor tissue [39]. Compared with VEL scope, Identafi is smaller and more convenient for use. However, some dysplasia, especially the early stage of dysplasia does not show fluorescence deficiency and excessive angiogenesis, which may lead to false negative results with VEL scope. Furthermore, the intensity of reflected light is not directly related to the risk degree of lesions. Hence it is difficult to determine the development stage of malignant progression based on the reflected light [40].

\section{Saliva Detection Techniques}

It is evident that molecular expression alternations are closely related to carcinogenesis. As saliva contains nucleic acids, proteins, metabolites and exfoliative cells, the genome, proteome and metabolites in it can provide biomarkers for the early diagnosis, malignant transformation detection and therapeutic response evaluation of OPMDs. In the last decades, various collection and extraction kits, such as DNA $\bullet \mathrm{SAL}^{\mathrm{Tm}}$ and RNAPro•SAL ${ }^{\mathrm{Tm}}$ (Oasis Diagnostics, Vancouver, WA, USA), have been applied to the extraction of DNA, RNA and proteins in saliva. The expression level of such molecules in saliva is usually 100 to 400 times lower than that in plasma, but they can also be detected through techniques with high detection sensitivity such as DNA microarray, miRNA-microarray, mass spectrometry and nanoscale biosensor, which makes saliva to be a diagnostic tool (Table 1). It is hoped that analysis of the genome, transcriptome, proteome and cellular metabolites will provide further insight into what happens at the subcellular level before clinically or histopathologically observable changes in OPMD. Salivary diagnosis is minimally invasive compared with oral tissue biopsy. However, the detection of a single marker is far insufficient to achieve a reliable result, which means the combination of some other biomarkers or detection tools is required for accurate diagnosis [41].

\subsection{Genomic Detection}

Carcinogenesis is usually accompanied with genetic alterations, such as loss of 
Table 1. Markers detection of saliva in OPMDs.

\begin{tabular}{|c|c|c|}
\hline Markers & $\begin{array}{c}\text { Observed change } \\
\text { (Dysplasia } V S \text { Normal) }\end{array}$ & References \\
\hline \multicolumn{3}{|l|}{ DNA } \\
\hline $\begin{array}{l}\text { Chromosome loci: } \\
3 p, 4 q, 8 p, 9 p, 11 q, 13 q, 17 p\end{array}$ & Loss of heterozygosity & (Zhang et al., 2012) \\
\hline $\begin{array}{l}\text { TP53 gene } \\
\text { Gene loci: p16, p14, MGMT, DAPK }\end{array}$ & $\begin{array}{l}\text { Mutation } \\
\text { Hypermethylation }\end{array}$ & $\begin{array}{l}\text { (Lindemann, et al., 2018) } \\
\text { (Carvalho et al., 2011; } \\
\text { Liu et al., 2012) }\end{array}$ \\
\hline \multicolumn{3}{|l|}{ miRNA } \\
\hline miRNA-21 & Overexpression & (Hung et al., 2016) \\
\hline miRNA-31 & Overexpression & (Hung et al., 2016) \\
\hline miRNA-146a & Overexpression & (Arao et al., 2012) \\
\hline miRNA-155 & Overexpression & ( Shi et al., 2015) \\
\hline miRNA-181b & Overexpression & (Cervigne et al., 2009) \\
\hline miRNA-184 & Overexpression & $\begin{array}{l}\text { (Zahran et al., 2015; } \\
\text { Cervigne et al., 2009) }\end{array}$ \\
\hline $\operatorname{miR}-208 b-3 p$ & Overexpression & (Fang et al., 2019) \\
\hline miRNA-345 & Overexpression & (Cervigne et al., 2009) \\
\hline miRNA-145 & Underexpression & (Zahran et al., 2015) \\
\hline miRNA-3065-5p & Underexpression & (Fang et al., 2019) \\
\hline \multicolumn{3}{|l|}{ Protein } \\
\hline Complement factor $\mathrm{H}(\mathrm{CFH})$ & Overexpression & (Chu et al., 2019) \\
\hline Fibrinogen alpha chain (FGA) & Overexpression & (Chu et al., 2019) \\
\hline Alpha-1-antitrypsin (SERPINAL 1) & Overexpression & (Chu et al., 2019) \\
\hline Interleukin-6 (IL-6) & Overexpression & (Khurshid et al., 2018) \\
\hline Interleukin-8 (IL-8) & Overexpression & (Elashoff et al., 2012) \\
\hline Tumor necrosis factor (TNF) & Overexpression & (Khurshid et al., 2018) \\
\hline Keratin-19 fragment (CYFRA21-1) & Overexpression & $\begin{array}{l}\text { (Khurshid et al., 2018; } \\
\text { Rajkumar et al., 2015) }\end{array}$ \\
\hline p53 protein & Overexpression & (Rosa et al., 2015) \\
\hline Ki-67 antigen & Overexpression & (Bienk Dias et al., 2017) \\
\hline Sialic acid & Overexpression & $\begin{array}{l}\text { (Joshi et al., 2010; } \\
\text { Dadhich et al., 2014) }\end{array}$ \\
\hline Reactive oxygen species (ROS) & Overproduction & $\begin{array}{l}\text { (Irimie et al., 2019; } \\
\text { Tvarijonaviciute et al., 2017) }\end{array}$ \\
\hline Reactive nitrogen species (RNS) & Overproduction & (Metgud et al., 2015) \\
\hline
\end{tabular}

heterozygosity (LOH), mutation, CNAs, and aberrant methylation. Polymerase chain reaction (PCR), DNA sequence and microarray are useful for genomic detection. $\mathrm{LOH}$ is known as a loss of genomic material in one of the chromosomal pairs, which is an early indicator of the OPMDs. $\mathrm{LOH}$ on chromosome arms $3 \mathrm{p}$, 
$9 p, 13 q$ and $17 p$ was highly correlated with carcinogenesis in oral cancer [42]. It was reported that $\mathrm{LOH}$ occurred at $3 \mathrm{p}$ and $9 \mathrm{p}$ appeared a 3.8 -fold risk of malignant transformation than the normal. Furthermore, individuals with $\mathrm{LOH}$ in $4 \mathrm{q}$, $8 \mathrm{p}, 11 \mathrm{q}$, or $17 \mathrm{p}$ had a 33 -fold increased carcinogenesis risk than the normal. Besides, the malignant risk degree of $\mathrm{LOH}$ occurred in $3 p$ or $9 \mathrm{p}$ showed a 22.6 -fold increase compared with the normal [43].

TP53 gene is a well-known tumor suppressor gene which inhibits cell cycle and initiates apoptosis in DNA damage. TP53 mutation is one of the most common genetic events occurred in the early stage of OPMDs malignant transformation [44]. DNA methylation is a common epigenetic modification, while aberrant DNA methylation could affect gene expression and the physiological stability of cell division. It's reported that hypermethylations occurred in p16, p14, MGMT and DAPK genes were highly correlated with carcinogenesis in premalignant oral lesions, indicating that aberrant DNA methylation could be a useful biomarker in malignant transformation and carcinogenesis of OPMDs [45] [46].

\section{2. miRNA Detection}

miRNAs are a class of short stable non-coding RNAs, which can regulate the expression levels of the target genes by binding to the 3 '-untranslated region (3'-UTR). Some of miRNAs are potential markers in malignant transformation and cancer. In the process of carcinogenesis, some miRNAs are upregulated, while others are downregulated, which can be analyzed by real-time quantitative polymerase chain reaction (RT-qPCR) and microarray techniques [47].

Studies showed that miRNA-21, miRNA-31, miRNA-146a, miRNA-155, miRNA-181b, miRNA-184, miR-208b-3p and miRNA-345 increased significantly in the saliva of malignant transformation of OPMDs, while miRNA-145 and miRNA -3065-5p appeared a downregulation trend [48]-[54]. Such miRNAs can be used as biomarkers for predicting malignancy of OPMDs to some extent. Once more data are obtained to analyze the miRNA expression of patients and a reference threshold of miRNA expression is developed, miRNA may be used as a reference index for OPMDs detection.

\subsection{Protein Detection}

Although numerous proteins in saliva have been reported as potential biomarkers, no one can be used as a direct and evident diagnostic indicator for OPMDs and OSCC. Thus, mass spectrometry and enzyme-linked immune sorbent assay (ELISA) technique is commonly used for proteinic quantitative analysis to provide auxiliary diagnostic approach for OPMDs.

Recently, it was found that the expression levels of complement factor $\mathrm{H}$ (CFH), fibrinogen alpha chain (FGA) and alpha-1-antitrypsin (SERPINAL1) were of great clinical significance to the conversion of OPMDs to OSCC [55]. Some studies showed that saliva proteins, such as interleukin-6 (IL-6), interleukin-8 (IL-8), tumor necrosis factor (TNF), keratin-19 fragment (CYFRA21-1), 
p53 protein and Ki-67 antigen, were overexpressed in OPMDs patients with malignant tendency. These proteins can be important molecular markers to diagnose OPMDs and predict the risk degree of OPMDs developing into OSCC [56] [57] [58] [59] [60].

\subsection{Sialic Acid Detection}

Sialic acid, also known as $\mathrm{N}$-acetylneuraminic acid, is a derivative of 9-carbon monosaccharide, serving as an important component of receptors on the surface of cell membrane. It could react with diphenylamine and its content can be quantitatively determined by spectrophotometer [61]. In OPMDs and OSCC, the level of sialic acid is found to increase due to the secretion, transformation and shedding of malignant cells at the early stage of carcinogenesis. It was reported that the sialic acid content in both OPMDs and OSCC was significantly higher than that in normal control. Moreover, the sialic acid content in OSCC was almost nine times more than that in OPMDs. It was also found that the average levels of salivary sialic acid in the control, OPMDs and oral cancer were 40.373, 57.562 and $80.422 \mathrm{mg} / \mathrm{dl}$, respectively, demonstrating a significant increase of sialic acid in OPMDs and oral cancer [62].

\subsection{Reactive Oxygen Species and Reactive Nitrogen Species Detection}

Reactive oxygen species (ROS) and reactive nitrogen species (RNS) are reactive substances and derivatives caused by cellular metabolism or exogenous carcinogens in the environment. Both of them cause oxidative damage to DNA and proteins, promote the formation of blood vessels and accelerate the invasion and metastasis of malignant cells. Once the intracellular balance between oxidation and antioxidation is broken, for instance, the oxidant increases and the antioxidant defense ability decreases in the patients of OPMDs, carcinogenesis may occur. ROS and RNS could play a role in assessing the risk of carcinogenesis analyzed by high performance liquid chromatography (HPLC) and colorimetric assay in patients with OPMDs [63].

Nitric oxide (NO), as a representative substance of RNS, is an important biological signal molecule. It was found that the salivary NO concentration in control group (median $=4.21 \mathrm{ug} / \mathrm{ml}$ ) was significantly lower than that in OPMDs group $($ median $=12.91 \mathrm{ug} / \mathrm{ml}, \mathrm{P}<0.001)$ [64]. Another study reported that ROS in oral lichen plaus (OLP) group was significantly increased compared with control group. Moreover, the levels of NO and nitrite in saliva of OLP patients were significantly higher than that in control group [65]. Thus, it seems evident that RNS could be a biomarker for the early diagnosis of OPMDs.

\section{Image Detection Techniques}

\subsection{Optical Coherence Tomography: OCT}

Optical coherence tomography (OCT) is a non-invasive, high-resolution optical 
imaging technique that captures images by measuring the intensity of light reflected by a target tissue. With a penetration depth of $1-3 \mathrm{~mm}$, OCT detects dysplasia by the fluctuation of light scattering due to cellular changes in dysplastic tissue in comparison to normal mucosa. This method is more accurate in detecting pathological tissue and can be used to roughly determine the edges and contours of OPMDs and OSCC [66]. It was believed that OCT can distinguish the mucosa with mild and moderate dysplasia by imaging the structures of the oral epithelial layer and lamina propria. The sensitivity, specificity, PPV and NPV of OCT for OPMDs and OSCC were $85 \%, 78 \%, 86.5 \%$ and $77.5 \%$, respectively. Therefore, OCT can be used as a simple auxiliary technique in the detection of OPMDs and OSCC [67].

\subsection{Reflectance Confocal Microscopy: RCM}

Reflective confocal microscopy (RCM) has been widely used in dermatology, and gradually applied to the observation of dental hard and soft tissue. As the refractive index of the detected components is different from that of the surrounding media, the microscopic details of the tissue and high-quality images for detection and diagnosis could be obtained by tomographic scanning, possibly with other techniques [68]. It works not only in detecting benign and malignant lesions, but also in identifying inflammatory reaction. Experimental results showed that RCM in vivo had a high consistency with histological detection. It has the potential as a non-invasive approach for the early detection of OPMDs and OSCC [69].

\section{Conclusion}

With the potential of malignant transformation, OPMDs may even develop into OSCC with poor prognosis, indicating that the surveillance of malignant progression for OPMDs may be the most effective way of oral cancer prevention. Since traditional approaches for the early diagnosis of OPMDs like tissue biopsy

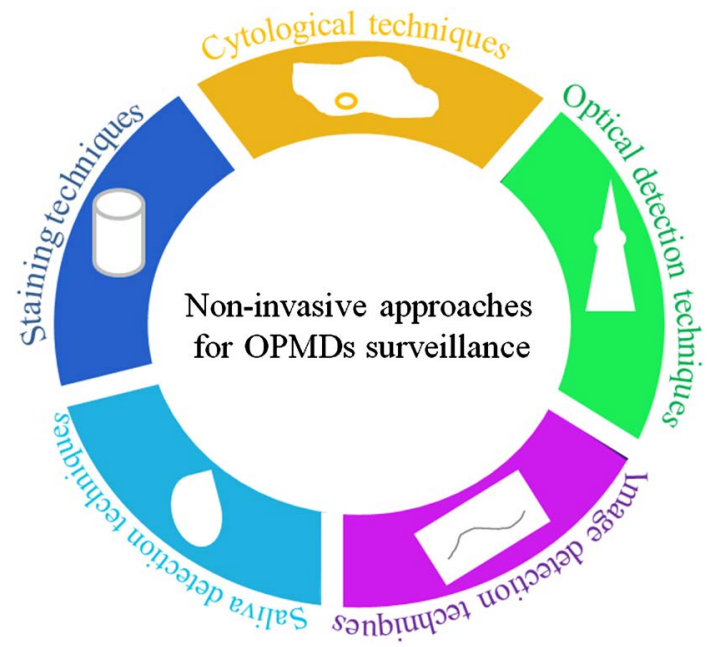

Figure 2. Non-invasive approaches for OPMDs surveillance. 
are invasive and cannot be repeated frequently, non-invasive techniques exhibit substantial advantages and have been applied in clinical practice (Figure 2). These techniques help to undertake oral mucosal screening, but additional levels of education including the level of knowledge and understanding of oral mucosal lesions do influence the effectiveness of the techniques. Besides, as extensively discussed above, each currently-available non-invasive approach shows its own advantages and drawbacks. It seems that a combination of such methods may overcome their limitations and improve detection accuracy for long-term disease surveillance of OPMDs.

\section{Acknowledgements}

This work was supported by National Natural Science Foundation of China (grant number 81901055).

\section{Conflicts of Interest}

The authors declare no conflicts of interest regarding the publication of this paper.

\section{References}

[1] Speight, P.M., Khurram, S.A. and Kujan, O. (2018) Oral Potentially Malignant Disorders: Risk of Progression to Malignancy. Oral Surgery, Oral Medicine, Oral Pathology and Oral Radiology, 125, 612-627. https://doi.org/10.1016/j.oooo.2017.12.011

[2] Chinn, S.B. and Myers, J.N. (2015) Oral Cavity Carcinoma: Current Management, Controversies, and Future Directions. Journal of Clinical Oncology, 33, 3269-3276. https://doi.org/10.1200/JCO.2015.61.2929

[3] Porter, S., Gueiros, L.A., Leao, J.C. and Fedele, S. (2018) Risk Factors and Etiopathogenesis of Potentially Premalignant Oral Epithelial Lesions. Oral Surgery, Oral Medicine, Oral Pathology and Oral Radiology, 125, 603-611. https://doi.org/10.1016/j.oooo.2018.03.008

[4] Messadi, D.V. (2013) Diagnostic Aids for Detection of Oral Precancerous Conditions. International Journal of Oral Science, 5, 59-65. https://doi.org/10.1038/ijos.2013.24

[5] Li, Y.N., Lu, R., Zhang, J. and Zhou, G. (2019) Inter- and Intra-Observer Agreement on the Judgment of Toluidine Blue Staining for Screening of Oral Potentially Malignant Disorders and Oral Cancer. Clinical Oral Investigations, 23, 1709-1714. https://doi.org/10.1007/s00784-018-2595-7

[6] Chainani-Wu, N., Madden, E., Cox, D., Sroussi, H., Epstein, J. and Silverman Jr., S. (2015) Toluidine Blue Aids in Detection of Dysplasia and Carcinoma in Suspicious Oral Lesions. Oral Diseases, 21, 879-885. https://doi.org/10.1111/odi.12358

[7] Awan, K., Yang, Y., Morgan, P. and Warnakulasuriya, S. (2012) Utility of Toluidine Blue as a Diagnostic Adjunct in the Detection of Potentially Malignant Disorders of the Oral Cavity_A Clinical and Histological Assessment. Oral Diseases, 18, 728-733. https://doi.org/10.1111/j.1601-0825.2012.01935.x

[8] Petruzzi, M., Lucchese, A., Baldoni, E., Grassi, F.R. and Serpico, R. (2010) Use of Lugol's Iodine in Oral Cancer Diagnosis: An Overview. Oral Oncology, 46, 811-813. 
https://doi.org/10.1016/j.oraloncology.2010.07.013

[9] Maeda, K., Suzuki, T., Ooyama, Y., Nakakuki, K., Yamashiro, M., Okada, N., et al. (2010) Colorimetric Analysis of Unstained Lesions Surrounding Oral Squamous Cell Carcinomas and Oral Potentially Malignant Disorders Using Iodine. International Journal of Oral \& Maxillofacial Surgery, 39, 486-492.

https://doi.org/10.1016/j.ijom.2009.11.001

[10] Nagaraju, K., Prasad, S. and Ashok, L. (2010) Diagnostic Efficiency of Toluidine Blue with Lugol's Iodine in Oral Premalignant and Malignant Lesions. Indian Journal of Dental Research, 21, 218-223. https://doi.org/10.4103/0970-9290.66633

[11] Murube, J. (2014) Rose Bengal: The Second Most Commonly Used Surfocular Vital Stain. The Ocular Surface, 12, 14-22. https://doi.org/10.1016/j.jtos.2013.10.003

[12] Du, G.F., Li, C.Z., Chen, H.Z., Chen, X.M., Xiao, Q., Cao, Z.G., et al. (2007) Rose Bengal Staining in Detection of Oral Precancerous and Malignant Lesions with Colorimetric Evaluation: A Pilot Study. International Journal of Cancer, 120, 1958-1963. https://doi.org/10.1002/ijc.22467

[13] Olms, C., Hix, N., Neumann, H., Yahiaoui-Doktor, M. and Remmerbach, T.W. (2018) Clinical Comparison of Liquid-Based and Conventional Cytology of Oral Brush Biopsies: A Randomized Controlled Trial. Head \& Face Medicine, 14, 9. https://doi.org/10.1186/s13005-018-0166-4

[14] Ye, X., Zhang, J., Tan, Y., Chen, G. and Zhou, G. (2015) Meta-Analysis of Two Computer-Assisted Screening Methods for Diagnosing Oral Precancer and Cancer. Oral Oncology, 51, 966-975. https://doi.org/10.1016/j.oraloncology.2015.09.002

[15] Kujan, O., Pemberton, M.N., Schwarz, M. and Sloan, P. (2018) Evaluation of an Innovative Oral Brush for Potential Applications Using Liquid Based Cytology. Journal of Oral Science, 60, 45-50. https://doi.org/10.2334/josnusd.16-0702

[16] Martini, M., Capodimonti, S., Cenci, T., Bilotta, M., Fadda, G., Larocca, L.M., et al. (2018) To Obtain More With Less: Cytologic Samples with Ancillary Molecular Techniques-The Useful Role of Liquid-Based Cytology. Archives of Pathology \& Laboratory Medicine, 142, 299-307. https://doi.org/10.5858/arpa.2017-0148-RA

[17] Alsarraf, A., Kujan, O. and Farah, C.S. (2018) Liquid-Based Oral Brush Cytology in the Diagnosis of Oral Leukoplakia Using a Modified Bethesda Cytology System. Journal of Oral Pathology \& Medicine, 47, 887-894. https://doi.org/10.1111/jop.12759

[18] Remmerbach, T.W., Pomjanski, N., Bauer, U. and Neumann, H. (2017) Liquid-Based versus Conventional Cytology of Oral Brush Biopsies: A Split-Sample Pilot Study. Clinical Oral Investigations, 21, 2493-2498.

https://doi.org/10.1007/s00784-017-2047-9

[19] Giaretti, W., Pentenero, M., Gandolfo, S. and Castagnola, P. (2012) Chromosomal Instability, Aneuploidy and Routine High-Resolution DNA Content Analysis in Oral Cancer Risk Evaluation. Future Oncology, 8, 1257-1271.

https://doi.org/10.2217/fon.12.116

[20] Alaizari, N.A., Sperandio, M., Odell, E.W., Peruzzo, D. and Al-Maweri, S.A. (2018) Meta-Analysis of the Predictive Value of DNA Aneuploidy in Malignant Transformation of Oral Potentially Malignant Disorders. Journal of Oral Pathology \& Medicine, 47, 97-103. https://doi.org/10.1111/jop.12603

[21] Chitturi, R.T., Nirmal, R.M., Sunil, P.M., Devy, A.S. and Reddy, B.V. (2014) Evaluation of Ploidy Status Using DNA-Image Cytometry of Exfoliated Mucosal Cells in Oral Lichen Planus. Journal of Cytology, 31, 131-135.

https://doi.org/10.4103/0970-9371.145629 
[22] Sun, Z., Liu, L., Wang, C., Qian, L., Yang, J., Zhao, Z., et al. (2019) Assessment of the Cancerization Risk for Oral Potentially Malignant Disorders by Clinical Risk Model Combined with Autofluorescence and Brush Biopsy with DNA-Image Cytometry. European Archives of Oto-Rhino-Laryngology, 276, 2549-2557. https://doi.org/10.1007/s00405-019-05520-7

[23] Ma, J.M., Zhou, T.J., Wang, R., Shan, J., Wu, Y.N., Song, X.L., et al. (2014) Brush Biopsy with DNA-Image Cytometry: A Useful and Noninvasive Method for Monitoring Malignant Transformation of Potentially Malignant Oral Disorders. European Archives of Oto-Rhino-Laryngology, 271, 3291-3295.

https://doi.org/10.1007/s00405-014-2935-4

[24] Xiao, X., Shi, L., Li, H., Song, Y., Liu, W. and Zhou, Z. (2015) DNA Content Status Using Brush Biopsy with Image Cytometry Correlated with Staging of Oral Leukoplakia: A Preliminary Study. Oral Oncology, 51, 59-63.

https://doi.org/10.1016/j.oraloncology.2014.10.009

[25] Castagnola, P., Zoppoli, G., Gandolfo, S., Monticone, M., Malacarne, D., Cirmena, G., et al. (2015) Genomic DNA Copy Number Aberrations, Histological Diagnosis, Oral Subsite and Aneuploidy in OPMDs/OSCCs. PLoS One, 10, e0142294. https://doi.org/10.1371/journal.pone.0142294

[26] Giaretti, W., Maffei, M., Pentenero, M., Scaruffi, P., Donadini, A., Di Nallo, E., et al. (2012) Genomic Aberrations in Normal Appearing Mucosa Fields Distal from Oral Potentially Malignant Lesions. Cellular Oncology, 35, 43-52. https://doi.org/10.1007/s13402-011-0064-2

[27] Salahshourifar, I., Vincent-Chong, V.K., Kallarakkal, T.G. and Zain, R.B. (2014) Genomic DNA Copy Number Alterations from Precursor Oral Lesions to Oral Squamous Cell Carcinoma. Oral Oncology, 50, 404-412. https://doi.org/10.1016/j.oraloncology.2014.02.005

[28] Shah, S.N., Manjunatha, B.S., Shah, V.S., Dagrus, K., Soni, N. and Shah, S. (2015) Quantitative Evaluation of Micronuclei in Oral Squamous Cell Carcinoma and Oral Submucous Fibrosis Patients: A Comparative Study. Recent Patents on Anti-Cancer Drug Discovery, 10, 233-238. https://doi.org/10.2174/1574892810666150317144817

[29] Juneja, S., Katyal, S., Rathore, A.S., Shetty, D.C., Tandon, A. and Jain, P. (2019) Utility of DNA-Specific Stains in Micronuclei Assay as a Marker of Genotoxicity in Oral Potentially Malignant Disorders and Oral Squamous Cell Carcinoma. Journal of Cytology, 36, 111-115. https://doi.org/10.4103/JOC.JOC_230_16

[30] Rashid, A. and Warnakulasuriya, S. (2015) The Use of Light-Based (Optical) Detection Systems as Adjuncts in the Detection of Oral Cancer and Oral Potentially Malignant Disorders: A Systematic Review. Journal of Oral Pathology \& Medicine, 44, 307-328. https://doi.org/10.1111/jop.12218

[31] Kammerer, P.W., Rahimi-Nedjat, R.K., Ziebart, T., Bemsch, A., Walter, C., Al-Nawas, B., et al. (2015) A Chemiluminescent Light System in Combination with toluidine Blue to Assess Suspicious Oral Lesions-Clinical Evaluation and Review of the Literature. Clinical Oral Investigations, 19, 459-466.

https://doi.org/10.1007/s00784-014-1252-Z

[32] Awan, K.H., Morgan, P.R. and Warnakulasuriya, S. (2015) Assessing the Accuracy of Autofluorescence, Chemiluminescence and Toluidine Blue as Diagnostic Tools for Oral Potentially Malignant Disorders-A Clinicopathological Evaluation. Clinical Oral Investigations, 19, 2267-2272. https://doi.org/10.1007/s00784-015-1457-9

[33] Awan, K.H., Morgan, P.R. and Warnakulasuriya, S. (2011) Utility of Chemiluminescence (ViziLite) in the Detection of Oral Potentially Malignant Disorders and 
Benign Keratoses. Journal of Oral Pathology \& Medicine, 40, 541-544. https://doi.org/10.1111/j.1600-0714.2011.01048.x

[34] Farah, C.S., Dost, F. and Do, L. (2019) Usefulness of Optical Fluorescence Imaging in Identification and Triaging of Oral Potentially Malignant Disorders: A Study of VEL Scope in the LESIONS Programme. Journal of Oral Pathology \& Medicine, 48, 581-587. https://doi.org/10.1111/jop.12896

[35] Tiwari, L., Kujan, O. and Farah, C.S. (2019) Optical Fluorescence Imaging in Oral Cancer and Potentially Malignant Disorders: A Systematic Review. Oral Diseases, 26, 491-510. https://doi.org/10.1111/odi.13071

[36] Simonato, L.E., Tomo, S., Scarparo Navarro, R. and Balbin Villaverde, A.G.J. (2019) Fluorescence Visualization Improves the Detection of Oral, Potentially Malignant, Disorders in Population Screening. Photodiagnosis and Photodynamic Therapy, 27, 74-78. https://doi.org/10.1016/j.pdpdt.2019.05.017

[37] Yamamoto, N., Kawaguchi, K., Fujihara, H., Hasebe, M., Kishi, Y., Yasukawa, M., et al. (2017) Detection Accuracy for Epithelial Dysplasia Using an Objective Autofluorescence Visualization Method Based on the Luminance Ratio. International Journal of Oral Science, 9, e2. https://doi.org/10.1038/ijos.2017.37

[38] Simonato, L.E., Tomo, S., Miyahara, G.I., Navarro, R.S. and Villaverde, A. (2017) Fluorescence Visualization Efficacy for Detecting Oral Lesions More Prone to Be Dysplastic and Potentially Malignant Disorders: A Pilot Study. Photodiagnosis and Photodynamic Therapy, 17, 1-4. https://doi.org/10.1016/j.pdpdt.2016.10.010

[39] Messadi, D.V., Younai, F.S., Liu, H.H., Guo, G. and Wang, C.Y. (2014) The Clinical Effectiveness of Reflectance Optical Spectroscopy for the in Vivo Diagnosis of Oral Lesions. International Journal of Oral Science, 6, 162-167. https://doi.org/10.1038/ijos.2014.39

[40] Lalla, Y., Matias, M.A. and Farah, C.S. (2016) Assessment of Oral Mucosal Lesions with Autofluorescence Imaging and Reflectance Spectroscopy. The Journal of the American Dental Association, 147, 650-660. https://doi.org/10.1016/j.adaj.2016.03.013

[41] Liu, D., Zhao, X., Zeng, X., Dan, H. and Chen, Q. (2016) Non-Invasive Techniques for Detection and Diagnosis of Oral Potentially Malignant Disorders. The Tohoku Journal of Experimental Medicine, 238, 165-177. https://doi.org/10.1620/tjem.238.165

[42] Fonseca-Silva, T., Diniz, M.G., de Sousa, S.F., Gomez, R.S. and Gomes, C.C. (2016) Association between Histopathological Features of Dysplasia in Oral Leukoplakia and Loss of Heterozygosity. Histopathology, 68, 456-460. https://doi.org/10.1111/his.12746

[43] Zhang, L., Poh, C.F., Williams, M., Laronde, D.M., Berean, K., Gardner, P.J., et al. (2012) Loss of Heterozygosity (LOH) Profiles-Validated Risk Predictors for Progression to Oral Cancer. Cancer Prevention Research (Phila), 5, 1081-1089. https://doi.org/10.1158/1940-6207.CAPR-12-0173

[44] Lindemann, A., Takahashi, H., Patel, A.A., Osman, A.A. and Myers, J.N. (2018) Targeting the DNA Damage Response in OSCC with TP53 Mutations. Journal of Dental Research, 97, 635-644. https://doi.org/10.1177/0022034518759068

[45] Carvalho, A.L., Henrique, R., Jeronimo, C., Nayak, C.S., Reddy, A.N., Hoque, M.O., et al. (2011) Detection of Promoter Hypermethylation in Salivary Rinses as a Biomarker for Head and Neck Squamous Cell Carcinoma Surveillance. Clinical Cancer Research, 17, 4782-4789. https://doi.org/10.1158/1078-0432.CCR-11-0324

[46] Liu, Y., Zhou, Z.T., He, Q.B. and Jiang, W.W. (2012) DAPK Promoter Hyperme- 
thylation in Tissues and Body Fluids of Oral Precancer Patients. Medical Oncology, 29, 729-733. https://doi.org/10.1007/s12032-011-9953-5

[47] El-Sakka, H., Kujan, O. and Farah, C.S. (2018) Assessing miRNAs Profile Expression as a Risk Stratification Biomarker in Oral Potentially Malignant Disorders: A Systematic Review. Oral Oncology, 77, 57-82. https://doi.org/10.1016/j.oraloncology.2017.11.021

[48] Cervigne, N.K., Reis, P.P., Machado, J., Sadikovic, B., Bradley, G., Galloni, N.N., et al. (2009) Identification of a microRNA Signature Associated with Progression of Leukoplakia to Oral Carcinoma. Human Molecular Genetics, 18, 4818-4829. https://doi.org/10.1093/hmg/ddp446

[49] Arao, T.C., Guimaraes, A.L., de Paula, A.M., Gomes, C.C. and Gomez, R.S. (2012) Increased miRNA-146a and miRNA-155 Expressions in Oral Lichen Planus. Archives of Dermatological Research, 304, 371-375. https://doi.org/10.1007/s00403-011-1197-x

[50] Yang, Y., Li, Y.X., Yang, X., Jiang, L., Zhou, Z.J. and Zhu, Y.Q. (2013) Progress Risk Assessment of Oral Premalignant Lesions with Saliva miRNA Analysis. BMC Cancer, 13, 129. https://doi.org/10.1186/1471-2407-13-129

[51] Shi, L.J., Zhang, C.Y., Zhou, Z.T., Ma, J.Y., Liu, Y., Bao, Z.X., et al. (2015) MicroRNA-155 in Oral Squamous Cell Carcinoma: Overexpression, Localization, and Prognostic Potential. Head Neck, 37, 970-976. https://doi.org/10.1002/hed.23700

[52] Zahran, F., Ghalwash, D., Shaker, O., Al-Johani, K. and Scully, C. (2015) Salivary microRNAs in Oral Cancer. Oral Diseases, 21, 739-747.

https://doi.org/10.1111/odi.12340

[53] Hung, K.F., Liu, C.J., Chiu, P.C., Lin, J.S., Chang, K.W., Shih, W.Y., et al. (2016) MicroRNA-31 Upregulation Predicts Increased Risk of Progression of Oral Potentially Malignant Disorder. Oral Oncology, 53, 42-47. https://doi.org/10.1016/j.oraloncology.2015.11.017

[54] Fang, C. and Li, Y. (2019) Prospective Applications of microRNAs in Oral Cancer. Oncology Letters, 18, 3974-3984. https://doi.org/10.3892/ol.2019.10751

[55] Chu, H.W., Chang, K.P., Hsu, C.W., Chang, I.Y., Liu, H.P., Chen, Y.T., et al. (2019) Identification of Salivary Biomarkers for Oral Cancer Detection with Untargeted and Targeted Quantitative Proteomics Approaches. Molecular \& Cellular Proteomics, 18, 1796-1806. https://doi.org/10.1074/mcp.RA119.001530

[56] Elashoff, D., Zhou, H., Reiss, J., Wang, J., Xiao, H., Henson, B., et al. (2012) Prevalidation of Salivary Biomarkers for Oral Cancer Detection. Cancer Epidemiology, Biomarkers \& Prevention, 21, 664-672. https://doi.org/10.1158/1055-9965.EPI-11-1093

[57] Rajkumar, K., Ramya, R., Nandhini, G., Rajashree, P., Ramesh Kumar, A. and Nirmala Anandan, S. (2015) Salivary and Serum Level of CYFRA 21-1 in Oral Precancer and Oral Squamous Cell Carcinoma. Oral Diseases, 21, 90-96. https://doi.org/10.1111/odi.12216

[58] Rosa, E.A., Lia, E.N., Macedo, S.B. and Amorim, R.F. (2015) In Situ Carcinoma Developed over Oral Lichen Planus: A Case Report with Analysis of BUB3, p16, p53, Ki67 and SOX4 Expression. Journal of Applied Oral Science, 23, 442-447. https://doi.org/10.1590/1678-775720150058

[59] Bienk Dias, K., Pereira Costa Flores, A., Gaiger Oliveira, M., Varvaki Rados, P. and Sant'ana Filho, M. (2017) Predictive Value of p63, ki-67, and Survivin Expression in Oral Leukoplakia: A Tissue Microarray Study. Microscopy Research and Technique, 80, 845-850. https://doi.org/10.1002/jemt.22872 
[60] Khurshid, Z., Zafar, M.S., Khan, R.S., Najeeb, S., Slowey, P.D. and Rehman, I.U. (2018) Role of Salivary Biomarkers in Oral Cancer Detection. Advances in Clinical Chemistry, 86, 23-70. https://doi.org/10.1016/bs.acc.2018.05.002

[61] Joshi, M. and Patil, R. (2010) Estimation and Comparative Study of Serum Total sialic Acid Levels as Tumor Markers in Oral Cancer and Precancer. Journal of Cancer Research and Therapeutics, 6, 263-266. https://doi.org/10.4103/0973-1482.73339

[62] Dadhich, M., Prabhu, V., Pai, V.R., D’Souza, J., Harish, S. and Jose, M. (2014) Serum and Salivary Sialic Acid as a Biomarker in Oral Potentially Malignant Disorders and Oral Cancer. Indian Journal of Cancer, 51, 214-218. https://doi.org/10.4103/0019-509X.146720

[63] Irimie, A.I., Braicu, C., Pasca, S., Magdo, L., Gulei, D., Cojocneanu, R., et al. (2019) Role of Key Micronutrients from Nutrigenetic and Nutrigenomic Perspectives in Cancer Prevention. Medicina (Kaunas), 55, 283. https://doi.org/10.3390/medicina55060283

[64] Metgud, R., Anandani, C. and Singh, K. (2015) Estimation of Salivary Nitric Oxide in oral Precancer Patients. Biotechnic \& Histochemistry, 90, 302-308. https://doi.org/10.3109/10520295.2014.998282

[65] Tvarijonaviciute, A., Aznar-Cayuela, C., Rubio, C.P., Ceron, J.J. and Lopez-Jornet, P. (2017) Evaluation of Salivary Oxidate Stress Biomarkers, Nitric Oxide and C-Reactive Protein in Patients with Oral Lichen Planus and Burning Mouth Syndrome. Journal of Oral Pathology \& Medicine, 46, 387-392. https://doi.org/10.1111/jop.12522

[66] Lee, C.K., Chi, T.T., Wu, C.T., Tsai, M.T., Chiang, C.P. and Yang, C.C. (2012) Diagnosis of Oral Precancer with Optical Coherence Tomography. Biomedical Optics Express, 3, 1632-1646. https://doi.org/10.1364/BOE.3.001632

[67] Hamdoon, Z., Jerjes, W., Upile, T., McKenzie, G., Jay, A. and Hopper, C. (2013) Optical Coherence Tomography in the Assessment of Suspicious Oral Lesions: An Immediate ex Vivo Study. Photodiagnosis and Photodynamic Therapy, 10, 17-27. https://doi.org/10.1016/j.pdpdt.2012.07.005

[68] Contaldo, M., Agozzino, M., Moscarella, E., Esposito, S., Serpico, R. and Ardigo, M. (2013) In Vivo Characterization of Healthy Oral Mucosa by Reflectance Confocal Microscopy: A Translational Research for Optical Biopsy. Ultrastructural Patholo$g y$, 37, 151-158. https://doi.org/10.3109/01913123.2013.776656

[69] Ardigo, M., Donadio, C., Franceschini, C., Catricala, C. and Agozzino, M. (2015) Interest of Reflectance Confocal Microscopy for Inflammatory Oral Mucosal Diseases. Journal of the European Academy of Dermatology and Venereology, 29, 1850-1853. https://doi.org/10.1111/jdv.12540 